\title{
Singularities in asymptotically anti-de Sitter spacetimes
}

\author{
Akihiro Ishibashi, ${ }^{1, *}$ and Kengo Maeda 2 , 田 \\ ${ }^{1}$ Department of Physics, Kinki University, Higashi-Osaka, 577-8502, Japan \\ ${ }^{2}$ Faculty of Engineering, Shibaura Institute of Technology, Saitama, 330-8570, Japan
}

(Dated: November 11, 2018)

\begin{abstract}
We consider singularity theorems in asymptotically anti-de Sitter (AdS) spacetimes. In the first part, we discuss the global methods used to show geodesic incompleteness and see that when the conditions imposed in Hawking and Penrose's singularity theorem are satisfied, a singularity must appear in asymptotically AdS spacetime. The recent observations of turbulent instability of asymptotically AdS spacetimes indicate that AdS spacetimes are generically singular even if a closed trapped surface, which is one of the main conditions of the Hawking and Penrose theorem, does not exist in the initial hypersurface. This may lead one to expect to obtain a singularity theorem without imposing the existence of a trapped set in asymptotically AdS spacetimes. This, however, does not appear to be the case. We consider, within the use of global methods, two such attempts and discuss difficulties in eliminating conditions concerning a trapped set from singularity theorems in asymptotically AdS spacetimes. Then in the second part, we restrict our attention to the specific case of spherically symmetric, perfect fluid systems in asymptotically AdS spacetime, and show that under a certain condition concerning dynamics of the fluid, a closed trapped surface must form, and as a combined result with Hawking and Penrose's theorem, that such a spacetime must be singular.
\end{abstract}

PACS numbers:

\section{INTRODUCTION}

Anti-de Sitter (AdS) spacetimes play a role of theoretical laboratory, where different areas of physics appear to make close contact, as exemplified in the AdS/CFT correspondence and its various applications [1]. In this context, it is essential, besides its asymptotic symmetries, that the conformal infinity of AdS spacetime is timelike and therefore can be viewed as another-lower dimensional - spacetime where non-gravitational theories dual to gravity theories in AdS are supposed to reside. AdS spacetimes are maximally symmetric, negatively curved, geodesically complete but non-globally hyperbolic, due to this timelike nature of infinity. As such, for any given point $p$ of AdS spacetime, all timelike geodesics from $p$ never reach the infinity and necessarily intersect the antipodal point $q$ to $p^{1}$. This can easily be seen by considering the cosmological chart

$$
d s^{2}=-d \tau^{2}+\cos ^{2} \tau\left(d \chi^{2}+\sinh ^{2} \chi d \Omega_{(D-2)}^{2}\right),
$$

where for simplicity the curvature radius is normalized to unity. This describes an open FLRW universe with bigbang and big-crunch respectively at $\tau \rightarrow \pm \pi / 2$, where the scale factor vanishes. In fact, because of its maximum isometries, for any given point $p$ in AdS, one can find the same cosmological chart in which $p$ corresponds to the big-bang or crunch point [see figure 1]. These big-bang/crunch points are merely a singular point of congruences of geodesic curves with the tangent $(\partial / \partial \tau)^{a}$ or a caustic thereof, but not a singularity of the spacetime structure itself. However, since all the comoving lines converge there, one can anticipate that if perturbed by, e.g., adding some matter fields, then the perturbation fields may grow along the comoving lines and eventually make big-bang/crunch points true curvature singularities. If this is the case it may be viewed also as a Cauchy horizon instability since the boundary of the causal past of $q$ is the Cauchy horizon for any $\tau=$ const. partial Cauchy surface.

To study such dynamics of perturbations in AdS spacetimes, suitable boundary conditions on perturbation fields need to be imposed at AdS infinity. It has been shown that under the Dirichlet (and some other types of) boundary conditions, AdS spacetime is stable at least with respect to linear perturbations 22. However, there has recently appeared evidence that asymptotically AdS spacetimes are non-linearly unstable 3, [4]. In particular, it has been shown by Bizon and Rostworowski [3] that upon certain assumptions, a black hole - hence singularities as well — must

\footnotetext{
*Electronic address: akihiro@phys.kindai.ac.jp

$\dagger$ Electronic address: maeda302@sic.shibaura-it.ac.jp

${ }^{1}$ Hereafter, by a $D$-dimensional AdS spacetime we mean the covering space of anti-de Sitter spacetime so that its topology is $\mathbb{R}^{D}$, rather than $S^{1} \times \mathbb{R}^{D-1}$, and therefore contains no closed timelike curves.
} 


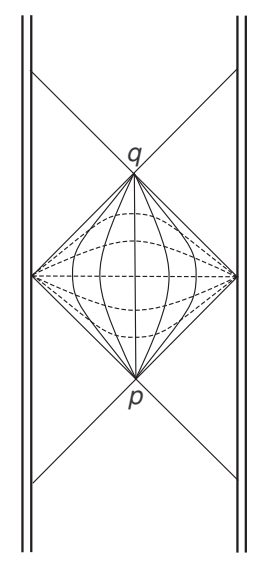

FIG. 1: The AdS-infinity is represented by a timelike surface. The diamond region is covered by an open FLRW chart. Every timelike geodesic through $p$ intersects all the timeslices defined by this chart and reach $q$, which corresponds to the anti-podal point to $p$.

be formed in asymptotically AdS spaces: They considered a spherically symmetric gravitating system of a minimally coupled massless scalar field and numerically shown that any Gaussian-type wave packet of the field does necessarily collapse to form a black hole.

This should be compared to the asymptotically flat case, in which it has been shown that small but non-linear perturbations remain small in its amplitude, and gravitational collapse of matter fields does not necessarily result in the black hole formation. More specifically, according to Choptuik's numerical calculation [5] of a scalar field collapse, the initial data set can be divided into two classes: supercritical and subcritical data. In the former case where the Gaussian-type wave packet has large amplitude, the scalar field collapses to form a black hole, whereas in the latter case, having small amplitude, the Gaussian wave packet bounces off the center of the spherical symmetry and escapes to infinity without forming a black hole. In contrast, the results of AdS nonlinear instability above [3] indicates that asymptotically AdS spacetimes are generically singular. One is therefore led to anticipate type of singularity theorems to be established in a class of asymptotically AdS spacetimes without there being any region of strong gravity to trap on initial data surface.

The purpose of this paper is to discuss singularity theorems in asymptotically AdS spacetimes. In the first part, we will attempt to clarify which results of the well-known singularity theorems can apply to the asymptotically AdS case. We briefly review the basics of singularity theorems and see that Hawking and Penrose's version of the singularity theorems applies to the AdS case without any major change in its assumptions, in section 【A The singularity theorems typically assume the existence of a strong gravity region, such as a closed trapped surface. However, the results of [3] indicates that a black hole forms even starting from arbitrarily small initial perturbations, and thus one may hope to obtain a singularity theorem with weaker, or even no, assumptions concerning strong gravity region. We discuss what the main obstacle is when attempting to eliminate the assumption of trapped sets from singularity theorems in asymptotically AdS case, in section ЩB In the second part, we will focus on a specific case: a system of spherically symmetric spacetime with a perfect fluid, and present a singularity theorem, in section III To show our singularity theorem we have to impose a condition that guarantees that the spacetime to consider must be dynamical in a certain sense. Also we will see that our proof need some restriction on the equation of state of the perfect fluid. We summarize our results in section IV and discuss future directions of the present subject. In Appendix, we discuss whether one can find a regular, static configuration in asymptotically AdS spacetime in a similar setting discussed in the main part of the paper.

\section{ASYMPTOTICALLY ANTI-DE SITTER SPACES AND SINGULARITIES}

\section{A. Hawking and Penrose's theorems and AdS spacetimes}

We start with briefly recapitulating basic ideas of the singularity theorems and then discuss whether (or which of) the singularity theorems can fit the case of asymptotically AdS spacetimes. A spacetime singularity is defined by, as one of its essential features, the causal geodesic incompleteness. The singularity theorems show that under 
physically reasonable conditions, the spacetime must be (either timelike or null, or both) geodesically incomplete. As "physically reasonable" conditions, typically imposed are

(a) The energy conditions

(b) The conditions on global structure,

(c) The existence of a strong gravity region

The condition (a) describes, via the Einstein equations, the convergence of causal geodesic congruences. For some versions of the singularity theorems, the generic conditions may also be required. The condition (b) is the requirement of either the strong causality or chronology condition or the existence of a Cauchy surface. The condition (c) requires a closed trapped surface for gravitational collapse, and in the cosmological context, a compact achronal hypersurface without edge or a point for which a null geodesic congruence starts to reconverge.

To show the geodesic incompleteness, the following two notions play a central role:

(i) Existence of (a pair of) conjugate points,

(ii) Global hyperbolicity.

The occurrence of a pair of conjugate points concerns the behavior of a geodesic congruence, which is governed by the Raychauduri equation:

$$
\frac{d \theta}{d s}=-R_{\mu \nu} K^{\mu} K^{\nu}-2 \sigma^{2}-\frac{1}{n} \theta^{2},
$$

where $s$ denotes an affine parameter of a geodesic curve with tangent $K^{\mu}, \theta$ the expansion of the geodesic congruence, $\sigma$ the shear, and the number $n$ depends on if $K^{\mu}$ is timelike or null and also on the number of spacetime dimensions $D$. At conjugate points, the expansion diverges: $\theta \rightarrow \pm \infty$. It is ensured by the condition (a) above - together with the generic condition, and is essentially due to the fact that gravity is attractive. The existence of conjugate points leads the following important result. Consider a future directed causal curve, $\gamma(t)$, from $p$ to $q \in J^{+}(p)$, whose length is given by

$$
L(\gamma: p \rightarrow q)=\int_{p}^{q} \sqrt{-g\left(\frac{\partial}{\partial t}, \frac{\partial}{\partial t}\right)} d t .
$$

Now suppose that $\gamma$ is geodesic and contains a point $r$ conjugate to $p$ between $p$ and $q$. Then one can find a future directed causal curve from $p$ to $q$ whose length is longer than $\gamma$, by considering the second variation of the length $L(\gamma: p \rightarrow q)$. A similar result holds between a point and a hypersurface.

A set $\mathcal{N}$ in $(M, g)$ is said to be globally hyperbolic if the strong causality holds on $\mathcal{N}$ and for any two points $p, q \in \mathcal{N}, J^{+}(p) \cap J^{-}(q)$ is compact, contained in $\mathcal{N}$. The importance of this notion for the singularity theorems is that it enables one to prove existence of maximal length causal curves. Namely, for any causally related two points, $p$ and $q \in J^{+}(p)$, in a globally hyperbolic region $\mathcal{N}$, there exists a geodesic curve from $p$ to $q$ in $\mathcal{N}$ which attains the maximal length among all continuous causal curves from $p$ to $q$ in $\mathcal{N}$. Again a similar result holds between a point and a hypersurface. This, combined together with the result from (i), implies that in a globally hyperbolic region $\mathcal{N}$, no causal geodesic curve connecting two points $p, q \in \mathcal{N}$ can contain a pair of conjugate points within the two points if it has the maximal length among all continuous causal curves from $p$ to $q$. The singularity theorems show that under suitable versions of the conditions (a), (b), and (c), if all geodesics are complete, then there must be a globally hyperbolic region within which every causal geodesic curve has to admit a pair of conjugate points on it, thus establishing a contradiction that falsifies the assumption of the geodesic completeness.

There are several different versions of the singularity theorems depending on different combinations of the conditions (a), (b), and (c). The first theorem [see Theorem 1 in chapter 8 of [6]], proposed by Penrose for gravitational collapse, requires the null convergence for the condition (a), the existence of a closed trapped surface as for (c), and the existence of non-compact Cauchy surface as for (b). The last one in particular requires that the entire spacetime $(M, g)$ be globally hyperbolic, which is clearly not the case for asymptotically anti-de Sitter spacetimes.

Hawking's theorem for cosmological singularities [see Theorem 4 in chapter 8 of [6]] removes the assumption of global hyperbolicity by, instead of requiring an expanding Cauchy surface, an expanding compact spacelike hypersurface without edge. AdS conformal boundary acts just like a boundary of a box that confines bulk fields. For this reason one might anticipate that Hawking's version of the singularity theorem may be generalized to apply to 
asymptotically AdS spacetimes. This theorem again does not straightforwardly apply as the AdS is not spatially compact $^{2}$.

A version which has much wider applicability is the one proposed by Hawking and Penrose [see Theorem 2 in chapter 8 of [6]], in which the condition (b) requires, instead of global hyperbolicity, the chronology condition and the condition (c) can be re-phrased as the existence of a future (or past) trapped set $\mathcal{S}$, an achronal set for which the horismos $E^{+}(\mathcal{S}):=J^{+}(\mathcal{S})-I^{+}(\mathcal{S})$ (or $E^{-}(\mathcal{S})$ ) is compact. Then, under the convergence and generic conditions to null geodesics, one can construct a globally hyperbolic region $\mathcal{N}$ which contains a future as well as past inextendible causal curve.

Now we argue that this version of the singularity theorems, which requires the existence of a closed trapped surface as a trapped set $\mathcal{S}$ required in (c), applies to the asymptotically AdS case without any major change in its assumptions, as far as the stress-energy tensor for matter fields together with the negative cosmological constant, does not violate the null, as well as the timelike convergence property. The point is that in asymptotically anti-de Sitter spacetimes the affine distance from any point in the spacetime to AdS boundary along a null geodesic is infinite, even though the coordinate distance may be finite. Therefore any outgoing null geodesics orthogonally emanating from the trapped surface, $\mathcal{S}$, must have a conjugate point with respect to $\mathcal{S}$ before reaching AdS-boundary, making the future horismos, $E^{+}(\mathcal{S})$, compact. Then, there is no obstruction to apply (Corollary of) Lemma 8.2.1 of [6] to show the existence of a future inextendible timelike curve $\gamma$ contained in the globally hyperbolic region $D^{+}\left(E^{+}(\mathcal{S})\right)$. Then, again using the fact that AdS-boundary is at affinely infinite distances along null geodesics from any point, we see that the past-inextendible extension of any null generating segment of $\dot{J}^{-}(\gamma)$ must enter $I^{-}(\gamma)$ and have a past end point, thus making $\mathcal{F}:=E^{+}(\mathcal{S}) \cap \bar{J}^{-}(\gamma)$ past-trapped: $E^{-}(\mathcal{F})$ is compact. Then, again applying Lemma 8.2.1 of [6], we also have a past inextendible timelike curve $\lambda$ in $D^{-}\left(E^{-}(\mathcal{F})\right)$. Note that to find the desired globally

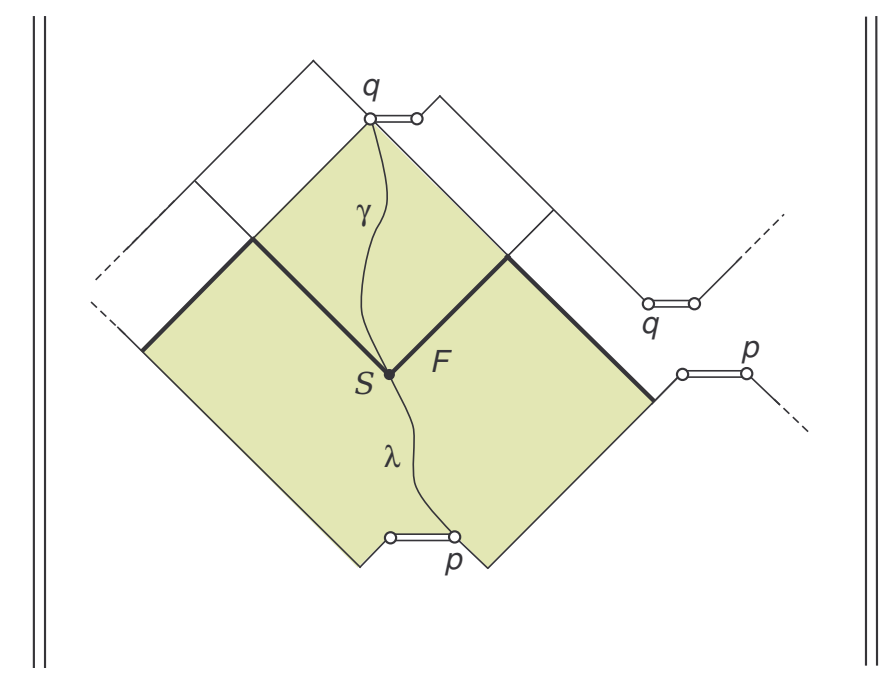

FIG. 2: $\mathcal{N}=D\left(E^{-}(\mathcal{F})\right.$ ) (Green color): To find this globally hyperbolic subregion in $M$, we have used the fact that in asymptotically anti-de Sitter spacetimes the affine distance from any point in the spacetime to AdS boundary along a null geodesic is infinite, as well as that the convergence property of null geodesics is not affected by the presence of the negative cosmological constant. Within this globally hyperbolic region, one can apply the argument about the maximum length curve.

hyperbolic region $\mathcal{N}=D\left(E^{-}(\mathcal{F})\right)$ we have used only the fact that in asymptotically anti-de Sitter spacetimes the affine distance from any point in the spacetime to AdS boundary along a null geodesic is infinite and that the convergence property of null geodesics is not affected by the presence of the negative cosmological constant. The rest

2 Note that if the magnitude of a negative cosmological constant $\Lambda$ does not dominate over other cosmic matter contents on some initial data surface, one might anticipate that either Penrose's or Hawking's theorem just mentioned above would apply since $\Lambda<0$ generally enhances the convergence of timelike geodesics and does not affect the convergence of null geodesics [as seen in (2.1) combined together with (2.3) below]. In fact, such a generalization of singularity theorems for open universes with a Cauchy surface or closed universes has been considered in the earlier work [7], in which the effects of a negative cosmological constant on the timelike convergence were taken into account. In that case, however, the requirement of either a Cauchy surface/global hyperbolicity or a compact surface without edge would not allow timelike conformal infinity to be attached, and therefore the entire spacetime would fail to be an asymptotically AdS spacetime considered in the present context. 
of arguments concerning the maximum length non-spacelike curves in the globally hyperbolic region int $\left(D\left(E^{-}(\mathcal{F})\right)\right)$ parallels the proof in Hawking and Ellis [6], but for later discussion, we shall sketch the arguments. Consider a sequence of points $x_{n}$ on $\lambda, x_{n+1} \in I^{-}\left(x_{n}\right)$, so that $\left\{x_{n}\right\}$ tends to the past as $n \rightarrow \infty$, and similarly a sequence of points $\left\{y_{n}\right\}$ on $\gamma$ tending to the future, $y_{n+1} \in I^{+}\left(y_{n}\right)$. Then as $x_{n}, y_{n}$ are in $\mathcal{N}$, one can find a timelike geodesic, $\mu_{n}$, from $x_{n}$ to $y_{n}$, which maximizes the length from $x_{n}$ to $y_{n}$. All $\mu_{n}$ cross the compact set $\mathcal{F}$, and therefore there exists a limit curve $\mu$ of $\left\{\mu_{n}\right\}$, which must also be a timelike geodesic contained within $\mathcal{N}$. Now if $\mu$ is incomplete, then we are done. If $\mu$ is complete, then $\mu$ must contain a pair of conjugate points due to the convergent condition (a) plus the timelike generic condition. This, however, implies that a timelike geodesic $\mu_{n}$ with sufficiently large $n$ would also have to admit a pair of conjugate points as conjugate points vary continuously. This is a contradiction against that $\mu_{n}$ was supposed to be the timelike curve of maximum length from $x_{n}$ to $y_{n}$.

Finally, we comment that the timelike convergence conditions assumed in the above arguments hold if, for any timelike vector $K^{\mu}$, the stress-energy tensor $T_{\mu \nu}$ for matter fields satisfies

$$
T_{\mu \nu} K^{\mu} K^{\nu} \geq \frac{1}{D-2}\left(T-\frac{1}{4 \pi} \Lambda\right) K_{\mu} K^{\mu}
$$

In fact, this is less restrictive than requiring $T_{\mu \nu}$ alone to satisfy the standard strong energy condition, due to the last term. In other words, the presence of $\Lambda<0$ enhances the timelike convergence $R_{\mu \nu} K^{\mu} K^{\nu} \geq 0$ via the Einstein equations.

Thus we have seen the following version of the singularity theorem applies to asymptotically AdS spacetimes:

Theorem. [Hawking and Penrose:]

A spacetime $\left(M, g_{\mu \nu}\right)$ is not timelike and null geodesically complete if the following conditions hold:

(a) $R_{\mu \nu} K^{\mu} K^{\nu} \geq 0$ for every non-spacelike vector $K^{\mu}$, and the generic condition holds for every non-spacelike geodesic, i.e., every non-spacelike geodesic has at least one point where $K_{[\lambda} R_{\nu] \alpha \beta[\mu} K_{\sigma]} K^{\alpha} K^{\beta} \neq 0$.

(b) The chronology condition holds.

(c) There exists a closed trapped surface.

Note that the condition (c) can be replaced with the other case: there exists a point for which light cones start reconverging.

\section{B. Difficulties in weakening the strong gravity conditions}

\section{Non-global hyperbolicity and maximum length of causal curves}

The non-linear instability of AdS spacetime from arbitrarily small initial perturbations [3, 4] indicates that singularities may generically form without there being any strong gravity region on initial data surface and thus motivates us to consider if one can obtain a singularity theorem without the condition for strong gravity. In this subsection we will discuss some difficulties in eliminating the condition (c).

The key point of the black hole formation from subcritical initial data in [3] is that the collapsing scalar field has to experience the process of bouncing-off at AdS infinity many times to make its configuration supercritical. This is not possible within a single, globally hyperbolic region such as the diamond region of the cosmological chart in figure1. Therefore, in order to obtain a singularity theorem without condition (c), one has to deal with a non-globally hyperbolic (sub)region. But then, the lack of global hyperbolicity falsifies standard arguments about the existence of maximum length geodesic curve in relevant region, which is one of the central ingredients of Hawking and Penrose's proof discussed in the previous section.

Let us consider this problem in a concrete example of pure AdS spacetime $(M, g)$. The AdS metric can be expressed by the standard global chart, $(t, \chi, \Omega)$, in the static form

$$
d s^{2}=\frac{1}{\cos \chi^{2}}\left(-d t^{2}+d \chi^{2}+\sin ^{2} \chi d \Omega^{2}\right)
$$

This is conformally embedded into the Einstein static universe $(\tilde{M}, \tilde{g})$, so that the conformal boundary $\partial M$ is attached to $M$ as $\tilde{M}=M \cup \partial M$, and located at $\chi= \pm \pi / 2$ in $\tilde{M}$. 
Now consider two points $p$ and $q$ which respectively correspond to the big-bang/crunch point in the open FLRW chart so that $p$ and $q$ form a pair of conjugate points. There are infinitely many timelike curves which maximize the length from $p$ to $q$, in fact all timelike geodesics emanating from $p$ reach the point $q$. Therefore no causal curves on $M$ contained in $J^{+}(p) \cap J^{-}(q)$ reaches the AdS conformal boundary. However, as mentioned just above, the black hole formation is not realized in a region which correspond to a single globally hyperbolic patch. Therefore, we are concerned with a pair of points $p$ and $q^{\prime}$, as depicted in Figure 3, for which $J^{+}(p) \cap J^{-}\left(q^{\prime}\right)$ contains a portion of $\partial M$. What we need to deal with is the causal curve that would maximize all causal curves connecting $p$ and $q^{\prime}$. It turns out that the timelike geodesic from $p$ to $q^{\prime}$ along the line $\chi=0$ is no longer the one. To see this, let us take a point $s$ on a null geodesic curve from $p$ to AdS infinity. Let $\chi_{s}$ be the coordinate value of $\chi$ at the point $s$. Then, move along $\chi_{s}=$ const. line, which is not a geodesic, until the point $r$ as in Figure 3 and move back to the center along a null geodesic to $q^{\prime}$. Thus we obtain a causal non-geodesic curve $p \rightarrow s \rightarrow r \rightarrow q^{\prime}$. One can easily evaluate the length of this path and show that this causal curve provides a longer length from $p$ to $q^{\prime}$ than the timelike geodesic along $\chi=0$. Since the null segment of this curve, those from $p$ to $s$ and $r$ to $q^{\prime}$, does not add any length to the curve, we only focus on the timelike segment of the $\chi_{s}$ line from $s$ to $r$. Then, since the length is given by eq. (2.2), we have

$$
L(s \rightarrow r) \propto \frac{1}{\cos \chi_{s}},
$$

which can become arbitrarily large as $\chi_{s} \rightarrow \pi / 2$. Thus, by taking the timelike segment $s \rightarrow r$ to be close to AdS conformal boundary, the length of the causal curve from $p \rightarrow r \rightarrow s \rightarrow q^{\prime}$ can be made arbitrarily large. This implies that there does not exist a causal curve that maximizes the length of all continuous causal curves from $p$ to $q^{\prime}$. Therefore even if one can show existence of inextendible causal curve in some region of AdS spacetime, one cannot apply the argument of a sequence of timelike geodesics $\mu_{n}$ to lead a contradiction.

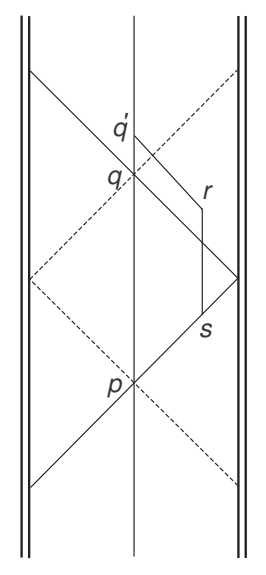

FIG. 3: In AdS spacetime, one can construct a causal curve from $p$ to $q^{\prime}$ which is not geodesic and has an arbitrarily large length.

Even in non-globally hyperbolic region, it might be possible to show the existence of maximum length curve between $p$ and $q^{\prime}$, if, for instance, one introduces a cut off radius $\chi_{c}$ so that any causal curves are not allowed to approach AdS conformal boundary beyond the cut-off coordinate value $\chi_{c}$. In that case, however, the maximum length curve would fail to be geodesic, and we would not be able to apply general results concerning variation of arc-length, established for geodesic curves in chapter 4 of [6].

\section{Double covering approach}

One way to avoid the issues that arise from the non-global hyperbolicity discussed above would be to consider a singularity theorem in some unphysical, globally hyperbolic spacetime, rather than in the physical asymptotically AdS spacetime itself. Namely, one attempts to

(1) construct a spatially compact unphysical spacetime from asymptotically AdS spacetime in a way that (i) the timelike convergence and (ii) the generic conditions are satisfied in the unphysical spacetime, and

(2) establish the relation between geodesic incompleteness in the unphysical spacetime and that in the physical asymptotically AdS spacetime. 
In fact, for a given asymptotically AdS spacetime $M$, one can first make a suitable conformal transformation to attach the boundary $\partial M$ to have $\tilde{M}=M \cup \partial M$, and then make double $\tilde{M}$ and glue them together at the AdS conformal boundaries $\partial M$. By doing so one can obtain a single connected spacetime $\widehat{M}$ with closed spatial section, just like the Einstein static cylinder, to which one may be able to apply type of the singularity theorems for closed universe. Since $\widehat{M}$ is spatially compact, any point or any subset $\mathcal{S} \subset \widehat{M}$ would be a trapped set, as $E^{ \pm}(\mathcal{S})$ is compact in $\widehat{M}$. Then, it is straightforward to show that under condition (a), the Cauchy horizon for $E^{ \pm}(\mathcal{S})$ must be non-compact or empty, and find a globally hyperbolic region $\widehat{\mathcal{N}}=D\left(E^{-}(\mathcal{F})\right)$ in $\widehat{M}$ that contains future and past inextendible timelike curve, say, $\gamma$. Then, one may hope to apply the argument of maximum length curve of timelike geodesics $\mu_{n}$ to show the geodesic incompleteness of $\widehat{M}$.

However, the following would be a potential obstruction in this approach. The double cover is taken from an unphysical spacetime whose metric is conformally isometric to the original, physical metric. Suppose one can find a suitable conformal transformation so that the timelike convergence conditions (2.3) are satisfied in unphysical spacetime. A subtle issue arises in the neighborhood of the AdS infinity $\partial M$ in $\widehat{M}$. Since we impose the asymptotic AdS boundary conditions at $\partial M$, we can find a neighborhood of $\partial M$ which is isometric to a neighborhood of the $\chi=\pi / 2$ line of the Einstein static universe. Then, it is straightforward to check that even on the neighborhood of the $\chi=\pi / 2$ line, the timelike convergence condition (2.3) is satisfied. However, there, the condition (2.3) is only marginally satisfied, and the generic condition is actually not. Hence the timelike geodesics that lie on the AdS boundary, i.e., those correspond to $\chi=\pi / 2$ lines, do not contain a pair of conjugate points. Therefore, even when one finds in $\widehat{\mathcal{N}}=D\left(E^{-}(\mathcal{F})\right) \subseteq \widehat{M}$, an inextendible causal curve $\mu$-which may be of the lines $\chi=\pi / 2$ itself-following the proof discussed in the previous section, one cannot apply the remaining arguments using the sequence of timelike geodesics $\mu_{n}$ to lead a contradiction, as $\mu$ would not contain a pair of conjugate points on it.

If we allow the AdS conformal boundary $\partial M$ to be dynamical and/or satisfy the generic condition in $\widehat{M}$ so that $\chi=\pi / 2$ lines or $\mu$ can contain a pair of conjugate points, then the double covering approach may work. In the case, however, the asymptotic symmetries in the original spacetime might possibly break down. [See e.g. [8] , for various possible boundary conditions in asymptotically AdS spacetimes.] It would be interesting to clarify possible dynamical boundary conditions on AdS conformal boundary that are generic enough so as to allow for the occurrence of a pair of conjugate points on every timelike geodesic in AdS conformal boundary, and at the same time, stringent enough so as to allow meaningful notion of asymptotic structure of AdS spacetimes.

\section{A SINGULARITY THEOREM IN SPHERICALLY SYMMETRIC SYSTEMS}

In the previous section $\amalg B$ we have seen difficulties in removing condition for strong gravity; without imposing the condition (c), the global method used in standard proof of the singularity theorems does not appear to work as we have to then deal with a non-globally hyperbolic region including part of AdS conformal infinity, in which the argument about the existence of maximum length causal curves does not apply. In this section we take a different approach to singularity theorems: we restrict our attention to a specific class of asymptotically AdS spacetimes and matter field. Since we have already seen that, once a trapped set is formed, then Hawking and Penrose's theorem applies, the main purpose here is to show the occurrence of a trapped set in asymptotically AdS spacetimes.

The numerical results of Bizon and Rostworowski [3] may be physically interpreted as follows. The dispersion of the scalar field increases during the time evolution and the amplitude tends to decrease when the wave packet travels toward the AdS boundary. However, near the AdS boundary, the effective potential makes the dispersion smaller by the reflection. Then, after several reflection, the wave packet can become supercritical near the center of spherical symmetry and finally collapses to a black hole.

Some basic aspects of gravitational collapse can be described by the Raychaudhuri equation for a congruence of non-spacelike curves. For example, let us consider an asymptotically AdS gravity system composed of a dust fluid with energy density $\mu>0$ and a negative cosmological constant $\Lambda<0$. Then, a congruence of timelike geodesics obeys the Raychaudhuri equation (2.1) with $R_{\mu \nu} K^{\mu} K^{\nu}=4 \pi \mu+|\Lambda|$. Since all the terms in the right-hand side are non-positive, the expansion $\theta$ becomes larger and larger in negative along the geodesics, and nothing stops the congruence getting smaller and smaller toward a conjugate point. This means that as the flow lines of the dust are timelike geodesics that also obey (2.1), the energy density blows up unboundedly toward the conjugate point, and a singularity eventually forms. It is clear from Eq. (2.1) that the negative cosmological constant plays a role to enhance the gravitational contraction.

This simple argument, however, does not immediately apply to the case with other matter models such as massless scalar fields or perfect fluids since the energy flow lines or the histories of small particles with non-zero pressure are not geodesic; they are accelerated. To be more precise, consider a perfect fluid in asymptotically AdS spacetime. Let 
$\mu$ and $p$ be the energy density and pressure of the fluid and let $V^{\mu}$ be the unit tangent vector field along the flow lines $(g(\boldsymbol{V}, \boldsymbol{V})=-1)$. Then, the Raychaudhuri equation of the congruence becomes

$$
\frac{d \theta}{d s}=-4 \pi(\mu+3 p)-2 \sigma^{2}-\frac{1}{3} \theta^{2}-|\Lambda|+\nabla_{\mu} \dot{V}^{\mu} .
$$

The first term is non-negative under the strong energy condition. The last term describing the divergence of the acceleration, $\dot{V}^{\mu}:=V^{\nu} \nabla_{\nu} V^{\mu}$, of the flow lines is related to the pressure gradient, as seen in Eq. (3.3b). In particular, the last term $\nabla_{\mu} \dot{V}^{\mu}$ is not necessarily negative and can represent repulsion between the fluid lines when it is positive. Therefore it is, in principle, possible that the last term and the others are balanced to realize an equilibrium, stationary configuration. It may be possible that even if the right-hand side is not always balanced and the expansion is negative at some initial time, it could change its sign to positive later on and the fluid lines would bounce off near the center and evolve outward. In this case, either the fluid bounces off at AdS boundary and recollapses to form singularities, or the system could be dynamical but admit a discrete time symmetry, i.e., it repeats expansion and contraction cyclically without ever forming singularities.

In the following we will show that for a certain class of matter fields, a singularity must inevitably form in spherically symmetric, asymptotically AdS spacetime. A key element of our argument is that as discussed above, the negative cosmological constant $\Lambda$ plays a role to effectively confine the system in an finite region characterized by its curvature radius. This, in turn, implies that the magnitude of the acceleration of the flow lines of matter fields should be bounded from above in certain sense so that the flow lines can never reach AdS infinity but instead must always reflect back inward the spacetime. ${ }^{3}$ Then, as can also be observed in Bizon and Rostworowski's massless scalar field system, we expect that even if the expansion of the matter flow lines can change its sign and gets positive, its time average over sufficiently large time interval (at least larger than the time scale specified by the cosmological constant) should be negative. If this is the case, then even though the flow lines of matter fields may have to bounce back and forth between the center of the spacetime and AdS-boundary repeatedly, a large part of the flow lines will eventually enter a compact region near the center with sufficiently small radius (compatible with the hoop conjecture) and form a closed trapped surface.

From different point of view, it is expected that such a singularity formation in asymptotically AdS spacetime occurs when the matter system under consideration admits no equilibrium configuration. The massless scalar field, for example, has no equilibrium configuration. On the other hand, charged fluid has an equilibrium configuration known as "electron stars" [9]. In the gravity system coupled to the charged fluid, dynamical instability will not occur, and hence the equilibrium configuration can be realized after the time evolution for any regular initial data except a particular case ${ }^{4}$. So, we will need to impose a kind of dynamical instability condition that ensures no equilibrium state to appear as an end state of the dynamical evolution.

In what follows we shall focus on spherically symmetric spacetimes with a negative cosmological constant $\Lambda<0$ and a perfect fluid with $\mu$ and $p$ being, respectively, the energy density and the pressure in four-dimensions. ${ }^{5}$ We assume that there is a regular, initial partial Cauchy surface whose topology is $\mathbf{R}^{3}$ as in the standard global AdS spacetime. A generalization of our arguments below to higher dimensions should be straightforward. We assume that our spaceitmes are asymptotically, globally anti-de Sitter, and the asymptotic fall off conditions for the perfect fluid are defined accordingly. ${ }^{6}$ These asymptotic conditions can be given in the conformal framework [See e.g., [10]]. For later convenience, we shall adopt the following coordinate system

$$
d s^{2}=-f(t, r) d t^{2}+h(t, r) d r^{2}+R^{2}(t, r) d \Omega^{2},
$$

where the time coordinate $t$ is taken along the fluid flow lines labelled by $r$, so that the unit future-directed tangent vector field is given by $V^{\mu}=f^{-1 / 2}(\partial / \partial t)^{\mu}$. Outside the support of the fluid, we assume that the time coordinate $t$ extends smoothly toward the AdS infinity at $r \rightarrow \infty$. Near the center $r=0$, due to the existence of the pressure, the timelike congruences of the fluid lines would never encounter a conjugate point, so that the coordinate system

3 For this we need to control the term $\nabla_{\mu} \dot{V}^{\mu}$ in eq. (3.1). If we assume, for instance, the local condition, $\nabla_{\mu} \dot{V}^{\mu}<|\Lambda|$, then we are immediately done. To show our singularity theorem we shall adopt a much weaker condition, [3.9], than this.

${ }^{4}$ For example, if initial spacelike hypersurface possesses a trapped surface, the time evolution results in the formation of singularity.

${ }^{5}$ Our argument does not work for the vacuum case with only a cosmological constant.

6 If a spherically symmetric configuration of the fluid has compact support on the initial partial Cauchy surface, then the outside of the support must be locally isometric to the Schwarzschild-AdS metric due to the Birkhoff's theorem, and therefore the event horizon and the central singularity must occur, unless a static configuration is realized. 
would not break down, as far as no singularities form. Assuming that $V^{\mu}$ is everywhere timelike and its orbits are complete, we shall show that a trapped region must form.

The perfect fluid under consideration obeys

$$
\begin{aligned}
& V^{\nu} \nabla_{\nu} \mu+(\mu+p) \nabla_{\nu} V^{\nu}=0, \\
& (\mu+p) \dot{V}^{\mu}+\left(g^{\mu \nu}+V^{\mu} V^{\nu}\right) \nabla_{\nu} p=0 .
\end{aligned}
$$

If we define $\rho$ as a number density of the fluid particles, $\rho$ satisfies

$$
\mu=\rho(1+\eta), \quad p=\rho^{2} \frac{d \eta}{d \rho},
$$

where $\eta$ is the internal energy. Then, substitution of Eq. (3.11) into these two equations yields

$$
\eta=A \rho^{\gamma-1}-1, \quad \mu=A \rho^{\gamma},
$$

where $A$ is a positive constant. The number current $j^{\mu}$ is also defined as $j^{\mu}:=\rho V^{\mu}$. So, we have a conservation law:

$$
\nabla_{\mu} j^{\mu}=0 .
$$

Since our main interest now on is the formation of a closed trapped surface in a spherically symmetric spacetime and thus concerns the behavior of the expansion $\theta$ of the fluid lines in the vicinity of the center, we shall consider below conditions that are required to hold for the fluid lines labelled by $0 \leq r<r_{0}$ with some positive (possibly sufficiently small) value $r_{0}$. At the same time, we are interested in situations in which the flow lines bounce off many times near the center by its pressure gradient and somewhere away from the center by the AdS curvature. Thus, we consider cases in which expansion of the fluid lines labelled by $r\left(<r_{0}\right)$ change many times its sign, minus for contraction and plus for expansion after bouncing off at the center. The first condition concerns geometric properties and the next two (ii), (iii) the dynamics, and (iv) the energy condition of the fluid.

\section{Condition (i):}

Let $X(t, r)$ collectively denote all physically relevant (gauge invariant) quantities such as $\theta, \nabla_{\mu} \dot{V}^{\mu}$, and $\mu$, and suppose $X$ be a smooth function of $r$ in the ball $0 \leq r<r_{*}$ with some relevant radius $r_{*}$. (a) Suppose there is a set of $t=$ constant hypersurfaces on which $|X(t, 0)| \geq K_{0}$ holds for any fixed positive number $K_{0}$. We require that there is a positive constant $r_{0}\left(<r_{*}\right)$, independent of $t$, such that for any $r$ in the range $0 \leq r \leq r_{0}$,

$$
|X(t, r)-X(t, 0)|<\frac{1}{2}|X(t, 0)| .
$$

(b) If, instead, $X\left(t, r_{i}\right)=0$ at $t=t_{i}^{\prime}$ for $r=r_{i}\left(0 \leq r_{i} \leq r_{0}\right)$, then we require that there is a positive constant $K$ (independent of $t_{i}^{\prime}$ ) such that for $\forall r ; 0 \leq r \leq r_{0}$,

$$
\left|X\left(t_{i}^{\prime}, r\right)\right|<K .
$$

As in condition (i), the expansion $\theta$ of $V^{\mu}$ is a smooth function of $t$ and $r$ in $0 \leq r \leq r_{0}$. Now one can view $\theta$ as a smooth function of proper time $s$ with fixed label $r\left(<r_{0}\right)$. Let $s_{1}$ be the proper time of the flow lines at which $\theta$ takes a local minimum (if exists) with $\theta\left(s_{1}\right)<0$ for the first time in the future of the initial surface, and similarly $s_{2}$ be such a time at which $\theta$ takes a local minimum with $\theta\left(s_{2}\right)<0$ for the first time after $s_{1}$, and so on. Then the following two cases can occur. Case (A): $\theta$ has only a finite number of local minima, or even does not admit a local minima from the beginning. Case (B): Such a local minimum of $\theta$ occurs infinitely many times. We require

\section{Condition (ii):}

In Case (A), there exist a time $t_{0}>0$ and a positive constant $c^{\prime}$ such that $\theta(t) \leq-c^{\prime}$ for $\forall t \geq t_{0}$ and $\forall r$ in $0 \leq r \leq r_{0}$ (i.e., $\theta$ does not increase if the minimum does not occur. See figure 4 ). In Case (B), there exists a positive constant $c$ such that for $\forall r$ in $0<r<r_{0}$ the following inequalities hold;

$$
\int_{s_{i}}^{s_{i+1}}\left(4 \pi(\mu+3 p)+2 \sigma^{2}+\frac{1}{3} \theta^{2}+|\Lambda|-\nabla_{\mu} \dot{V}^{\mu}\right) d s \geq c .
$$

Physically this condition implies that the gravitational contraction effect is stronger than the effect of the repulsion by pressure gradient in a sense of "time average." In what follows we call this condition the averaged convergence 


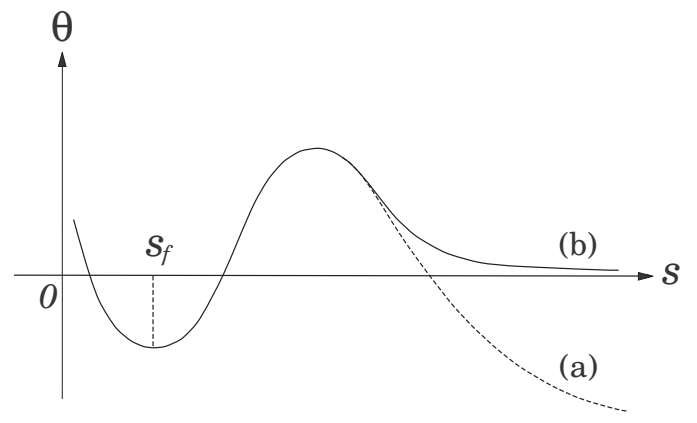

FIG. 4: Two typical cases regarding the condition (ii) are shown in Case (A): $s_{f}$ is the time when $\theta$ has its local minimum last. The condition (ii) is satisfied in the branch (a), while it is violated in the branch (b).

condition. For the case in which this condition is not satisfied, one may be able to find an equilibrium, static configuration of matter field in asymptotically AdS spacetimes. In Appendix, we discuss such a static configuration cannot be realized for perfect fluid satisfying certain equation of state.

\section{Condition (iii):}

Given a hypersurface $t=t(r)$, consider, on $t=t(r)$, the proper radial length $l\left(r_{0}\right)$ from the center to $r_{0}$ and the area radius $R\left(t, r_{0}\right)$ of the spherical shell labelled by $r_{0}$. We require that $l\left(r_{0}\right)$ and $R\left(t, r_{0}\right)$ be bounded above by some positive constants;

$$
R\left(t, r_{0}\right) \leq R_{0}, \quad l\left(r_{0}\right):=\int_{0}^{r_{0}} \sqrt{h^{2}(t(\tilde{r}), \tilde{r})-f(t(\tilde{r}), \tilde{r}) t^{2}(\tilde{r})} d \tilde{r} \leq l_{0}
$$

where $R_{0}$ and $l_{0}$ denote some time-independent positive constants.

This condition comes from the role of the AdS boundary that confines the system in a finite region.

\section{Condition (iv):}

The equation of state of perfect fluid satisfies the following; $\mu>0$ and

$$
p=(\gamma-1) \mu, \quad 1<\gamma<2 .
$$

Now we wish to establish the following theorem:

\section{Theorem.}

If the conditions (i)-(iv) are satisfied, then a future trapped surface necessarily forms in the future of the initial surface.

Once the occurrence of a closed trapped surface, $\mathcal{S}$, is shown ${ }^{7}$, the Hawking-Penrose theorem discussed in section $\llbracket \mathrm{A}$ can be applied. We have

\section{Corollary.}

If the conditions (i)-(iv) are satisfied, then any spherically symmetric, asymptotically AdS spacetime with perfect fluid is not timelike or null geodesically complete.

Note that the case $\gamma=1$ in condition (3.11) corresponds to a spherically symmetric dust fluid in asymptotically AdS spacetime. The dynamics of such a spacetime is described by the Lemaitre-Tolman-Bondi metric with cosmological

\footnotetext{
7 It is worth mentioning the numerical work 12] showing that a (globally) naked singularity appears in the perfect fluid when $1<\gamma \leq$ 1.0105 in asymptotically flat case. At first look, even though the calculation of [12] does not include cosmological constant, our lemma may appear to contradict the result of [12] because we show the existence of a trapped surface, indicating that the resulting singularity is enclosed by the event horizon. However, we are assuming here that the timelike congruences near the center are future complete (The proper time is supposed to be extendible at least until that far future that a closed trapped surface is formed). So, this indicates that for some initial data with $\gamma \leq 1.0105$, a timelike congruence is incomplete and a central singularity inevitably appears before a trapped surface would form.
} 
constant $\Lambda$. In this case, the last term in the Raychaudhuri equation (3.1) disappears. In this case it has been shown that either black holes or naked singularities form as the end state of the dust collapse. [see e.g. 11] and references therein]. Therefore in the following we focus on the $1<\gamma<2$ case.

Proof.) We first note that under the required conditions, in particular, condition (ii), we have either one of the following cases. Case (A): the expansion $\theta$ of the tangent vector $V^{\mu}$ - viewed as a function of $t$ with fixed $r\left(<r_{0}\right)$ admits local minima only a finite number of times and afterward behaves monotonically with $t$, or the local minimum never occurs in the future of initial surface. Case (B): the local minima occur infinite number of times (on the assumption, of course, of geodesic completeness, that is, the spacetime under consideration could be extended as far future as one wishes).

We consider these two cases separately. Let us start with Case (A) that a local minimum disappears within finite times. By condition (ii), $\theta(t, r)<-c<0$ for $t \geq t_{0}$ and $0 \leq r \leq r_{0}$. Define a volume $V\left(t, r_{0}\right)$ enclosed by $r=r_{0}$ ball at $t=$ const. spacelike hypersurface:

$$
V\left(t, r_{0}\right):=\int_{0}^{r_{0}} v(t, r) d r d \Omega
$$

where $v=\sqrt{h} R^{2}$ and the expansion $\theta$ is rewritten by $v$ as

$$
\theta=\frac{1}{v} \frac{d v}{d s} .
$$

Since $\theta(t, r)<-c$ for $0 \leq \forall r \leq r_{0}$, the volume element $v$ at any $r \leq r_{0}$ approaches zero, implying $V \rightarrow 0$ at $s \rightarrow \infty$. Now take a maximal surface $\Sigma$ (that is, a surface whose extrinsic curvature has the vanishing trace) in the future of $t=t_{*}\left(t_{*} \rightarrow \infty\right)$ const. hypersurface. The unit normal future-directed vector $\xi$ on $\Sigma$ and unit outward pointing vector $\eta$ normal to two-sphere of fixed radius in $\Sigma$ are written by

$$
\xi^{\mu}=V^{\mu} \cosh \alpha+n^{\mu} \sinh \alpha, \quad \eta^{\mu}=V^{\mu} \sinh \alpha+n^{\mu} \cosh \alpha,
$$

where the boost parameter $\alpha$ is taken to be positive (so that $\sinh \alpha>0$ ) and where $n$ is a unit normal outward vector on $t=$ const. hypersurface, i. e. , $g(V, n)=0, g(n, n)=1$. Let us define the energy density $\hat{\mu}$ and the current $\hat{\pi}$ on $\Sigma$ as

$$
\hat{\mu}:=T_{\mu \nu} \xi^{\mu} \xi^{\nu}-\frac{\Lambda}{8 \pi}, \quad \hat{\pi}=-\frac{1}{8 \pi} R_{\mu \nu} \xi^{\mu} \eta^{\nu}=-T_{\mu \nu} \xi^{\mu} \eta^{\nu} .
$$

Note that here we define $T_{\mu \nu}$ so as to include a negative cosmological constant term $-\Lambda g_{\mu \nu} / 8 \pi$ and therefore $T_{\mu \nu}$ above is slightly different from that in (2.3). Then, $\hat{\mu}-\hat{\pi}$ is calculated as

$$
\hat{\mu}(t(r), r)-\hat{\pi}(t(r), r)=\{\sinh \alpha(\sinh \alpha+\cosh \alpha) \gamma+1\} \mu(t(r), r),
$$

where $t=t(r)\left(>t_{*}\right)$ on $\Sigma$.

By the conservation law (3.6), we have

$$
\begin{aligned}
& \mathcal{N}\left(r_{0}\right)=\int_{\Omega} \int_{0}^{r_{0}} v\left(t_{*}, r\right) \rho(t, r) d r d \Omega \\
& =\left.\int_{\Omega} \int_{0}^{r_{0}} \rho(t(r), r) \cosh \alpha(t(r), r) d \hat{V}\right|_{\Sigma} \\
& =\int_{\Omega} \int_{0}^{r_{0}} \rho(t(r), r) v(t(r), r) d r d \Omega,
\end{aligned}
$$

where $d \hat{V}$ is the volume element on $\Sigma$. Since $v(t(r), r)$ goes to zero for $0 \leq r \leq r_{0}$ in the limit $t_{*} \rightarrow \infty, \rho(t(r), r)$ diverges in the limit. Owing to the relation $\mu=A \rho^{\gamma}($ (3.5) $), \mu(t(r), r)$ also diverges in the limit.

Following Ref. [13], we shall define the total material energy $E$ and the radial material momentum $P$ enclosed by $r=r_{0}$ shell on the hypersurface $\Sigma$ as

$$
(E, P):=\int_{\Omega} \int_{0}^{r_{0}}\left(\hat{\mu}(t(r), r)+\frac{\Lambda}{8 \pi}, \hat{\pi}(t(r), r)\right) d \hat{V}
$$


Then, by Eq. (3.16), we have

$$
\begin{aligned}
E-P & =\int_{\Omega} \int_{0}^{r_{0}}\left\{\hat{\mu}(t(r), r)+\frac{\Lambda}{8 \pi}-\hat{\pi}(t(r), r)\right\} d \hat{V} \\
& =\int_{\Omega} \int_{0}^{r_{0}}\left\{(\sinh \alpha(\sinh \alpha+\cosh \alpha) \gamma+1) \mu(t(r), r)+\frac{\Lambda}{8 \pi}\right\} d \hat{V} \\
& =4 \pi \int_{0}^{r_{0}}\left\{\frac{(\sinh \alpha(\sinh \alpha+\cosh \alpha) \gamma+1)}{\cosh \alpha} \mu(t(r), r)+\frac{\Lambda}{8 \pi \cosh \alpha}\right\} v(t(r), r) d r \\
& \geq 4 \pi \int_{0}^{r_{0}}\left(\mu(t(r), r)+\frac{\Lambda}{8 \pi}\right) v(t(r), r) d r \\
& =4 \pi \int_{0}^{r_{0}}\left(A \rho^{\gamma}(t(r), r)+\frac{\Lambda}{8 \pi}\right) v(t(r), r) d r \\
& >4 \pi \int_{0}^{r_{0}}\left(A \rho(t(r), r)+\frac{\Lambda}{8 \pi}\right) v(t(r), r) d r \\
& \geq A \mathcal{N}\left(r_{0}\right)+\frac{\Lambda}{8 \pi} V\left(t_{*}, r_{0}\right) .
\end{aligned}
$$

Here, we have used the fact that

$$
\sinh \alpha(\sinh \alpha+\cosh \alpha) \gamma+1 \geq \cosh \alpha, \quad \rho^{\gamma}(t(r), r)>\rho(t(r), r), \quad v(t(r), r)<v\left(t_{*}, r\right) .
$$

Since $V\left(t_{*}, r_{0}\right) \rightarrow 0$ for $t_{*} \rightarrow \infty$ and $\rho(t(r), r) \rightarrow \infty$ for $0 \leq \forall r \leq r_{0}$ when $t_{*} \rightarrow \infty$ and $\gamma>1$, the dominant energy condition should be satisfied for $0 \leq r \leq r_{0}$ and $E-P$ should also diverge when $t_{*} \rightarrow \infty$. By the condition (iii), the proper radial length $l\left(r_{0}\right)$ along the maximal surface is bounded from above, implying

$$
E-P \geq l\left(r_{0}\right)
$$

when $t_{*} \rightarrow \infty$. According to Ref. [13], this yields a future apparent horizon, due to the condensation of positive energy enclosed by the small ball $0 \leq r \leq r_{0}$ on the maximum surface. Thus, Case (A) follows.

We turn to Case (B), i.e., when the minimum occurs infinite times. Integrating the Raychaudhuri equation

$$
\frac{d \theta}{d s}=-R_{\mu \nu} V^{\mu} V^{\nu}-2 \sigma^{2}-\frac{1}{3} \theta^{2}+\nabla_{\mu} \dot{V}^{\mu},
$$

we immediately obtain

$$
\theta_{s_{i+1}}-\theta_{s_{i}}<-c
$$

from the condition (ii). Then, repeating this $N^{\prime}$ times, we finally obtain

$$
\theta_{s_{N^{\prime}}}<-N^{\prime} c+\theta_{s_{0}} \text {. }
$$

From this, it follows that for any positive integer $N$, one can always find $t_{i}$ such that

$$
\theta\left(t_{i}\right)<-N c,
$$

for $0 \leq r \leq r_{0}$.

Note that (3.25) implies that the amplitude of the expansion (at least) grows linearly in time. This is in accord with the appearance of secular terms in non-linear perturbations of the gravity system coupled to a massless scalar field [3] or the pure gravity system in non-spherically symmetric asymptotically AdS spacetime [4].

Next, integrating Eq. (3.22) for a small spatial interval $[r, r+\Delta r]$ and a small time interval $\left[t_{N}, t_{N}+\Delta t\right]$, we obtain

$$
\begin{aligned}
4 \pi\left(\left.\theta(r) \sqrt{h} R\right|_{s_{N}(r)+\Delta s(r)}-\left.\theta(r) \sqrt{h} R\right|_{s_{N}(r)}\right) \Delta r & =-4 \pi \int_{s_{N}(r)}^{s_{N}(r)+\Delta s(r)} \sqrt{h} R\left(R_{\mu \nu} V^{\mu} V^{\nu}+2 \sigma^{2}+\frac{1}{3} \theta^{2}\right) d s \Delta r \\
& +\left.4 \pi \int_{s_{N}(r)}^{s_{N}(r)+\Delta s(r)} R \dot{V}^{\mu} n_{\mu} d s\right|_{r+\Delta r}-\left.4 \pi \int_{s_{N}(r)}^{s_{N}(r)+\Delta s(r)} R \dot{V}^{\mu} n_{\mu} d s\right|_{r}
\end{aligned}
$$


where $t=t_{N}$ is the time when the expansion $\theta$ at the center takes minimum $\Theta_{N}$, and $s_{N}(r), \Delta s(r)$ are the proper time at $t=t_{N}$, the proper time interval between $\left[t_{N}, t_{N}+\Delta t\right]$ of each $r=$ const. timelike curve, respectively. To derive this equation, we have used $V_{\mu} \dot{V}^{\mu}=0$.

Since $\theta$ at the center takes minimum $\Theta_{N}$ at $t=t_{N}, \theta$ begins to increase from $t=t_{N}$. This implies, by (3.25), that

$$
\left.\dot{V}^{\mu} n_{\mu}\right|_{\Delta r, t=t_{N}}-\left.\dot{V}^{\mu} n_{\mu}\right|_{r=0, t=t_{N}}>\frac{(N c)^{2}}{3} \sqrt{h} \Delta r,
$$

By the condition (i), one also obtains

$$
\left.\dot{V}^{\mu} n_{\mu}\right|_{r+\Delta r, t_{N}}-\left.\dot{V}^{\mu} n_{\mu}\right|_{r, t_{N}}>\left.\frac{\epsilon(N c)^{2}}{3} \sqrt{h} \Delta r\right|_{r, t_{N}}
$$

for any $r$ in the range $0 \leq r \leq r_{0}$, where $\epsilon$ is a positive value $1 / 2<\epsilon<1$.

Now consider $t=t_{N}$ spacelike hypersurface. Using a spatial proper radius $l$ on the hypersurface, Eq. (3.3b) is rewritten as

$$
(\mu+p) \dot{V}^{\mu} n_{\mu}=-p_{, l} .
$$

Substituting Eq. (3.11) into this equation, we obtain

$$
\dot{V}^{\mu} n_{\mu}=-\frac{\gamma-1}{\gamma \mu} \mu_{, l} .
$$

Then, the inequality (3.28) is reduced to

$$
-\left.\frac{(\gamma-1) \mu, l}{\gamma \mu}\right|_{l+\Delta l}+\left.\frac{(\gamma-1) \mu_{, l}}{\gamma \mu}\right|_{l}>\frac{\epsilon(N c)^{2}}{3} \Delta l
$$

for $0 \leq l \leq l_{0}$, where $l_{0}=l\left(r_{0}\right)$. Taking the limit $\Delta l \rightarrow 0$, we finally obtain

$$
\left(\frac{(\gamma-1) \mu_{, l}}{\gamma \mu}\right)_{, l}<-\frac{\epsilon(N c)^{2}}{3} \text {. }
$$

From the regularity at the center $r=0, \mu_{, l}$ must be zero at the center. So, integrating the inequality (3.32) from $l=0$ to $l$, one gets

$$
\frac{\mu_{, l}}{\mu} \leq-\frac{N l}{l_{d}^{2}}
$$

where $l_{d}:=\sqrt{3(\gamma-1) / \epsilon \gamma c^{2} N}$. Further integration yields

$$
\mu(l) \leq \mu(0) \exp \left[-\left(N / 2 l_{d}^{2}\right) l^{2}\right] .
$$

Since we are considering Case (B), we can take an arbitrary large integer $N$, and therefore the above inequality means that the dispersion gets small indefinitely, as $t_{N}$, hence $l_{d}^{-1}$, gets large. As seen in Eq. (3.34), most of the energy exists inside $l<l_{d}$ for large $N$. Since $\rho\left(l_{d}\right) \ll \rho(0)$, we can conclude that $l\left(r=r_{0}\right)$ should be inside $l_{d}$ by the condition (i). As $t_{N} \rightarrow \infty, l_{d} \rightarrow 0$, and $l\left(r_{0}\right)$ also goes to zero. Thus, it is clear that the proper spatial volume $V\left(t_{N}, r_{0}\right)$ enclosed by $r=r_{0}$ on $t=t_{N}$ spacelike hypersurface also goes to zero by the condition (iii) because

$$
V\left(t_{N}, r_{0}\right)=4 \pi \int_{0}^{l\left(r_{0}\right)} R^{2}(l) d l \leq 4 \pi \int_{0}^{l\left(r_{0}\right)} R_{0}^{2} d l=4 \pi R_{0}^{2} l\left(r_{0}\right) \rightarrow 0 .
$$

This means that $\mu(0)=A \rho^{\gamma}(0)$ diverges due to the conservation law (3.6).

Having seen that $\mu$ can become arbitrarily large near the center, we also see that the curvature near the center becomes arbitrarily large. Since the congruence begins to expand between $t_{N}<t<t_{N+1}$ due to the pressure, there is a time $t_{N}^{\prime}\left(>t_{N}\right)$ when the expansion $\theta$ among the congruence between $0 \leq r \leq r_{0}$ first becomes zero. For later 
purpose, let us say that $\theta\left(t_{N}\right)$ becomes zero first at $r=r_{i}\left(0<r_{i}<r_{0}\right)$. Now consider the Hamiltonian constraint equation at $t=t_{N}^{\prime}$ spacelike hypersurface. The constraint equation is

$$
\begin{aligned}
\mathcal{R} & =2 \Lambda+16 \pi \mu-\left(\chi_{a}^{a}\right)^{2}+\chi^{a b} \chi_{a b} \\
& >16 \pi \mu+2 \Lambda-\theta^{2},
\end{aligned}
$$

where $\mathcal{R}$ is the scalar curvature of the hypersurface and $\chi_{a b}$ is the second fundamental form. To derive the inequality, we have used the fact that $\chi^{a b} \chi_{a b}$ is positive and $\chi_{a}^{a}=\theta$.

Since $\theta$ is non-positive among the congruences between $0 \leq r \leq r_{0}$ during $t_{N} \leq t \leq t_{N}^{\prime}$, the volume $V\left(t_{N}^{\prime}, r_{0}\right)$ enclosed by $r=r_{0}$ on $t=t_{N}^{\prime}$ spacelike hypersurface is smaller than the volume $V\left(t_{N}, r_{0}\right)$. Since $V\left(t_{N}, r_{0}\right)$ goes to zero, $V\left(t_{N}^{\prime}, r_{0}\right)$ also goes to zero when $N \rightarrow \infty$. Thus, we obtain $\mu\left(t_{N}^{\prime}, r\right) \geq \mu\left(t_{N}, r\right)$ for $r$ in $0 \leq r \leq r_{0}$. Since a finite mass is concentrated in the small neighborhood of the symmetry center, one can expect that a trapped surface, $\mathcal{S}$, would form, enclosing the mass. We will show below this is indeed the case.

Let us take the metric on the $t=t_{N}^{\prime}$ spacelike hypersurface as

$$
d s_{3}^{2}=\frac{d R^{2}}{k(R)}+R^{2} d \Omega^{2} .
$$

Then, as shown in Ref. [14], a marginal surface for which the expansion $\theta_{+}$of the outgoing null geodesics becomes zero appears only when $k(R)=0$. Namely, in terms of the local mass $M(R)$ defined as $k(R)=1-2 M(R) / R$, the marginal surface appears only when

$$
\frac{2 M(R)}{R}=1
$$

Note that $M(0)=0$ by imposing regularity at the center.

In the coordinates (3.37), the scalar curvature $\mathcal{R}$ is calculated as

$$
\mathcal{R}=\frac{4 M^{\prime}(R)}{R^{2}}
$$

Substituting Eq. (3.39) into Eq. (3.36) and integrating once, we obtain

$$
M(r)>4 \pi \int_{0}^{R(r)} \mu(r) R^{2} d R-\int_{0}^{R(r)}\left(\frac{|\Lambda|}{2}+\frac{\theta^{2}}{4}\right) R^{2} d R .
$$

Since $\theta\left(t_{N}^{\prime}, r\right)=0$ at $r=r_{i}^{\prime}\left(0 \leq r_{i}^{\prime} \leq r_{0}\right),\left|\theta\left(t_{N}^{\prime}, r\right)\right|<K$ in $0 \leq r \leq r_{0}$ by the condition (i). This implies that the second term in the r.h.s. of Eq. (3.40) is negligible compared with the first term, and hence $M(r) \geq 0$ for $0 \leq r \leq r_{0}$. Thus, it is clear that $R\left(r_{0}\right)$ goes to zero when $V\left(t_{N}^{\prime}, r_{0}\right)$ goes to zero because

$$
V\left(t_{N}^{\prime}, r_{0}\right)=4 \pi \int_{0}^{r_{0}} \frac{R^{2}}{1-\frac{2 M(R)}{R}} d R>\frac{4 \pi}{3} R^{3}\left(r_{0}\right) .
$$

By the conservation law (3.6), we have

$$
4 \pi \int_{0}^{l\left(r_{0}\right)} \rho(R(l)) R^{2}(l) d l=\mathcal{N},
$$

where $l\left(r_{0}\right)$ is the proper radial length from $r=0$ to $r=r_{0}$. Since $l\left(r_{0}\right) \leq l_{0}$ by the condition (iii),

$$
\rho(R) \geq \frac{C_{2}}{R^{2}\left(r_{0}\right)}
$$

for $0 \leq r \leq r_{0}$ by the condition (ii), where $C_{2}$ is some positive constant. By the relation $\mu(R)=A \rho^{\gamma}(R)$, we also obtain

$$
\mu(R) \geq \frac{C_{3}}{R^{2 \gamma}\left(r_{0}\right)}
$$


where $C_{3}$ is a positive constant. Substituting Eq. (3.44) into Eq. (3.40), we obtain

$$
M\left(r_{0}\right) \geq \frac{4 \pi C_{4}}{3} R^{3-2 \gamma}\left(r_{0}\right),
$$

where $C_{4}$ is a positive constant. Thus, we have

$$
\lim _{N \rightarrow \infty} \frac{2 M\left(r_{0}\right)}{R\left(r_{0}\right)}=\lim _{N \rightarrow \infty} \frac{8 \pi C_{4}}{3} R^{2(1-\gamma)}\left(r_{0}\right)>1,
$$

where we have used the fact $R\left(r_{0}\right) \rightarrow 0$ when $N \rightarrow \infty$ and the condition (iv). This means that $k(R)$ must admit zero, hence there must appear a trapped surface.

\section{CONCLUDING REMARKS}

In this paper, we have considered singularity theorems in asymptotically AdS spacetimes. We first briefly reviewed the basics of singularity theorems and checked that Hawking and Penrose's theorems, which use the global methods to prove, apply to asymptotically AdS spacetimes without major changes in its assumptions. Motivated from the result of [3] that indicates that in a spherically symmetric massless scalar field model, a black hole inevitably forms even starting from arbitrarily small initial perturbations after repeating the reflection at AdS infinity many times, we have discussed if it is possible to show a singularity theorem in asymptotically AdS spacetimes by taking into account the effect of the reflection back at AdS infinity, and instead removing the requirement of a strong gravity region, i.e., a trapped set, which is one of the essential requirements in Hawking and Penrose's singularity theorems. We have discussed main obstacles to do so in section

Then in the second part we have focused on the system of asymptotically AdS gravity plus a perfect fluid with spherical symmetry and have investigated under what conditions a closed trapped surface generically appears in such a system. As seen in section 【A once a closed trapped surface forms in asymptotically AdS spacetime, the existence of a singularity can be shown under conditions used in Hawking-Penrose singularity theorems. We have shown that if the "averaged convergence condition" introduced in section III satisfied, a trapped surface necessarily forms, provided that the proper time $s$ of the fluid lines are complete, or the spacetime can be extended as far future from initial data as one wants. Apart from that, there could be the case in which a singularity occurs without forming a closed trapped surface. In this case singularities that occur would be a naked one. Thus, we conclude that a singularity generically appears in the spherically symmetric asymptotically AdS spacetime for the perfect fluid system considered in section III]

The "averaged convergence condition" implies that the expansion of congruence for the fluid world lines changes its sign infinitely many times and the absolute value at each local minimum continues to increase as the spacetime dynamically evolves. As shown in section III this makes the dispersion of the energy density profile smaller and smaller indefinitely. It is noteworthy that imposing the "averaged convergence condition" does not necessarily mean by itself the existence of any strong gravity region or a high energy density region. In this sense, the condition is much weaker than the condition for the existence of a closed trapped surface, where a sufficiently large amount of mass should be enclosed in a small compact region.

It would be interesting to generalize our results here for the perfect fluid system to other matter field cases such as a massless or massive scalar field. To define the "averaged convergence condition" for the scalar field case, we need to define the notion of the energy flow lines of a scalar field. Kodama vector field [15], which can be defined for any spherically symmetric spacetimes, should be a candidate of such a vector field because, associated with it, one can always construct a conserved current.

It would be also interesting to explore under what circumstances, singularities are avoided to form in asymptotically AdS spacetime. At first sight, all known numerical and analytical results [3, 4] combined with our results suggest that any matter fields satisfying physically reasonable energy conditions inevitably cause singularity formation in asymptotically AdS spacetime. According to the AdS/CFT correspondence, this phenomenon can be interpreted as a turbulent instability in hydromechanics, which transfers energy to higher frequency, as mentioned in Refs. [3, 4]. On the other hand, it is well-known in the turbulent phenomena that there is also an "inverse cascade phenomenon" which transfers energy to lower frequency. In the gravity side, if repulsive force is much stronger than attractive force for a matter field, such an inverse cascade phenomenon might occur since singularity formation would be avoided due to the large repulsive force. To answer this question, it would be worth investigating a charged perfect fluid in which the charge density is much bigger than the mass density. If the repulsive force is stronger than the attractive force, a periodic solution might be possible, as the charged fluid cannot escape to AdS infinity or collapse to a singularity. 
[See e.g. [16] for a different approach to the problem.] To pursue these possibilities, we need to investigate, by concerted efforts of both numerical and analytical methods, non-linear dynamics for a variety of matter fields in asymptotically AdS spacetimes.

\section{Acknowledgments}

We wish to thank Vitor Cardoso, Oscar Dias, Jorge Santos, Helvi Witek for valuable discussions. We would especially like to thank Gary T. Horowitz for valuable discussions, in particular, on the non-existence of maximum length curve and the need of cut-off radius discussed in section IB as well as on the occurrence of a closed trapped surface in the last part of section III. We would also like to thank Tomohiro Harada for discussions on numerical studies of the singularity formation by perfect fluids and Tetsuya Shiromizu for useful comments on the first version of the paper. The authors thank the Yukawa Institute for Theoretical Physics at Kyoto University. Discussions during the YITP workshop, YITP-T-11-08 on "Recent advances in numerical and analytical methods for black hole dynamics" were very useful to complete this work. This work was supported in part (AI) by the Grant-in-Aid for Scientific Research Fund of the JSPS (C)No. 22540299 and by the Barcelona Supercomputing Center (BSC) under Grant No. AECT-2012-2-0005 and in part (KM) by MEXT/JSPS KAKENHI Grant Number 23740200.

\section{Appendix: Non-equilibrium state}

Let us check if there is a static regular asymptotically AdS solution in the case of the perfect fluid under consideration. If the system admits a static configuration, dynamically perturbed solutions would approach the static equilibrium configuration, unless the perturbation is unstable. In this case, the condition (ii) would be violated, and the expansion $\theta$ approaches zero at future infinity (case (b) in figure 4). As shown below, however, when $\gamma>3 / 2$, this is not the case.

As in Ref. [6], we shall adopt the following coordinate system

$$
d s^{2}=-\frac{d t^{2}}{F^{2}(r)}+X^{2}(r) d r^{2}+r^{2} d \Omega^{2} .
$$

In the case of perfect fluid with the energy-momentum tensor:

$$
T_{\mu \nu}=\mu V_{\mu} V_{\nu}+\left(g_{\mu \nu}+V_{\mu} V_{\nu}\right) p,
$$

the Einstein equations are written

$$
\begin{aligned}
& 4 \pi(\mu+p)=\frac{X^{\prime}}{r X^{3}}-\frac{F^{\prime}}{r F X^{2}}, \\
& 8 \pi p=\Lambda-\frac{1}{r^{2}}\left(1-\frac{1}{X^{2}}\right)-\frac{2 F^{\prime}}{r F X^{2}}, \\
& 4 \pi(\mu-p)=-\Lambda+\frac{1}{r^{2}}+\frac{F^{\prime}}{r F X^{2}}+\frac{1}{r^{2} X^{3}}\left(r X^{\prime}-X\right) .
\end{aligned}
$$

By eliminating $p$ from Eqs. 4.3a and 4.3b), we obtain

$$
8 \pi \mu=-\Lambda+\frac{1}{r^{2}}\left(1-\frac{1}{X^{2}}\right)+\frac{2 X^{\prime}}{r X^{3}} .
$$

Defining the mass $m(r)$ as

$$
X^{2}=\left(1-\frac{2 m}{r}-\frac{\Lambda}{3} r^{2}\right)^{-1}
$$

$m(r)$ is obtained from Eq. (4.4) as

$$
m(r)=4 \pi \int_{0}^{r} \mu(\tilde{r}) \tilde{r}^{2} d \tilde{r},
$$

where the regularity condition $m(0)=0$ is imposed. 
By the conservation law of the energy-momentum tensor (4.2), we have

$$
p^{\prime}=(\mu+p) \frac{F^{\prime}}{F} .
$$

Substitution of Eqs. (4.5) and (4.7) into Eq. (4.3b) yields

$$
p^{\prime}=-(\mu+p) \frac{m+\left(4 \pi p-\frac{\Lambda}{3}\right) r^{3}}{r\left(r-2 m-\frac{\Lambda}{3} r^{3}\right)} .
$$

Using Eq. (3.11), we finally obtain the evolution equation for $\mu$ as

$$
(\gamma-1) \mu^{\prime}=-\frac{\gamma \mu\left(m+4 \pi(\gamma-1) \mu r^{3}-\frac{\Lambda}{3} r^{3}\right)}{r\left(r-2 m-\frac{\Lambda}{3} r^{3}\right)} .
$$

We can determine the metric functions $X$ and $F$ via Eqs. (4.5) and (4.7) by solving Eqs. (4.9) and (4.6) by imposing the regularity condition at the center, $\mu^{\prime}(0)=0$. So, for a fixed $\Lambda$ and $\gamma$ the only free parameter is $\mu(0)$. By Eq. (4.9), we can easily check that there is no star like solution such as

$$
\mu>0 \quad \text { for } \quad r<R \quad \text { and } \quad \mu=0 \quad \text { for } \quad r \geq R,
$$

Suppose the existence of the solution satisfying Eq. (4.10). Then, just inside of $R, r<R$, Eq. (4.9) behaves as $\mu^{\prime} \sim-C \mu$, where $C$ is a positive constant. The solution is $\mu \sim A e^{-C r}$ for some constant $A$, which fails to satisfy Eq. (4.10). This indicates that the energy density $\mu$ decays toward the infinity but never becomes zero at any finite radius $R$.

The asymptotic behavior of $\mu$ is easily obtained by Eq. (4.9). Since $\mu$ decays toward the infinity, $\Lambda$ term in Eq. (4.9) is dominant at $r \rightarrow \infty$. So, Eq. (4.9) is reduced to

$$
(\gamma-1) \mu^{\prime} \simeq-\frac{\gamma \mu}{r}
$$

with the asymptotic solution $\mu \sim r^{-\frac{\gamma}{\gamma-1}}$. If we impose a boundary condition at the infinity as $m \sim$ const., we must take $\gamma$ as

$$
\gamma<\frac{3}{2}
$$

This implies that any static configuration satisfying asymptotically AdS spacetime cannot be realized when

$$
\frac{3}{2} \leq \gamma<2 .
$$

[1] J. Maldacena, "The Large N limit of superconformal field theories and supergravity" Adv. Theor. Math. Phys. 2, 231-252 (1998); O. Aharony, S. Gubser, J. Maldacena, H. Ooguri, and Y. Oz, "Large N field theories, string theory and gravity" Phys. Rep. 323, 183-386 (2000).

[2] A. Ishibashi and R. M. Wald, "Dynamics in nonglobally hyperbolic static space-times. 3. Anti-de Sitter space-time," Class. Quant. Grav. 21, 2981 (2004) hep-th/0402184.

[3] P. Bizon and A. Rostworowski, "Weakly Turbulent Instability of Anti-De Sitter Spacetime," Phys. Rev. Lett. 107, 031102 (2011)

[4] O. J. C. Dias, G. T. Horowitz, and J. E. Santos, "Gravitational Turbulent Instability of Anti-de Sitter Space," arXiv:1109.1825[hep-th].

[5] M. W. Choptuik, "Universality and scaling in gravitational collapse of a massless scalar field," Phys. Rev. Lett. 709 (1993).

[6] S. W. Hawking and G. F. R. Ellis, The large scale structure of space-time. Cambridge: Cambridge University Press, 1973

[7] F.J. Tipler, "Singularities in universes with negative cosmological constant" Astrophys. J. 209, 12-15 (1976)

[8] T. Hertog and K. Maeda, "Black holes with scalar hair and asymptotics in $N=8$ supergravity" JHEP 0407051 (2004)

[9] S. A. Hartnoll and A. Tavanfar, "Electron stars for holographic metallic criticality," Phys. Rev. D. 83 046003 (2011).

[10] S. Hollands, A. Ishibashi and D. Marolf, "Comparison between various notions of conserved charges in asymptotically AdS-spacetimes" Class. Quant. Grav. 22, 2881-2920 (2005) 
[11] S. S. Deshingkar, S. Jhingan, A. Chamorro, and P. S. Joshi, "Gravitational collapse and cosmological constant" Phys. Rev. D 63, 124005 (2001)

[12] T. Harada, "Final fate of the spherically symmetric collapse of a perfect fluid", Phys. Rev. D. 58104015 (1998).

[13] J. Guven and N. O. Murchadha, "Sufficient conditions for apparent horizons in spherically symmetric initial data" Phys. Rev. D. 567658 (1997)

[14] S. A. Hayward, "Gravitational Energy in Spherical Symmetry" Phys. Rev. D. 531938 (1996).

[15] H. Kodama, "Conserved Energy Flux for the Spherically Symmetric System and the Backreaction Problem in the Black Hole Evaporation" Prog. Theor. Phys. 63 (1980) 1217

[16] T. Shiromizu, S. Ohashi, and R. Suzuki, "A no-go on strictly stationary spacetimes in four/higher dimensions" arXiv:1207.7250 gr-qc]. 